\title{
Circuit
}

Musiques contemporaines

CIRCUIT

\section{Identités dans un monde de pluralité : musique(s) et société(s) au tournant du $\mathrm{XXI}^{\mathrm{e}}$ siècle \\ Identity in a world of plurality: music(s) and society(ies) at the turn of the twenty-first century}

\section{Noémie Pascal}

Volume 15, numéro 2, 2005

Cartes d'identités

URI : https://id.erudit.org/iderudit/902356ar

DOI : https://doi.org/10.7202/902356ar

Aller au sommaire du numéro

Éditeur(s)

Les Presses de l'Université de Montréal

ISSN

1183-1693 (imprimé)

1488-9692 (numérique)

Découvrir la revue

Citer cet article

Pascal, N. (2005). Identités dans un monde de pluralité : musique(s) et société(s) au tournant du XXI ${ }^{\mathrm{e}}$ siècle. Circuit, 15(2), 55-72.

https://doi.org/10.7202/902356ar

\section{Résumé de l'article}

Cet article s'attarde à la problématique de l'identité, concept lié à l'unité et l'unicité, dans un monde musical marqué par un esprit de pluralité. En effet, l'expérience de la musique des dix dernières années à travers les festivals internationaux de musique contemporaine laisse voir avant tout un éclatement généralisé qui favorise particulièrement les questionnements identitaires, tant d'un point de vue personnel (identité du compositeur) que social (identité nationale). Or, la recherche d'identité sociale et d'identité personnelle en musique peuvent-elles réellement cohabiter? Est-il possible de trouver l'identité? donc l'unité? d'une oeuvre, d'un compositeur, d'une nation, au-delà de la diversité qui les habite? L'article ouvre de multiples pistes de réflexion d'ordre tantôt ontologique, tantôt sociologique, en posant des questions qui concernent à la fois l'oeuvre musicale d'aujourd'hui, son créateur, son auditeur, et le monde dans lequel ils évoluent. 


\section{Identités dans un monde de pluralité : musique(s) et société(s) au tournant du XXI ${ }^{e}$ siècle}

Noémie Pascal

Il est probablement utopique d'essayer de définir l'identité de la musique «savante» d'aujourd'hui dans son ensemble : elle est elle-même divisée en de multiples genres, courants, esthétiques, sous des «ismes » avoués ou rejetés qui traversent sans obstacle les frontières géographiques. Pourtant, si nombreuses que soient ces tendances, toutes appartiennent à la même époque, empreintes de préoccupations communes : elles sont autant de détails d'un même tableau.

Face à cette fragmentation, il est assurément plus aisé de se prononcer sur l'identité d'une parcelle bien délimitée de ces musiques, comme le font les discours musicologiques sur l'identité nationale ou sur l'appartenance de certaines œuvres à des courants esthétiques donnés, plutôt que sur leur globalité, comme cet article propose de le faire. Car en visant un questionnement identitaire global, nous sommes vite placés devant une complexité remplie d'ambiguités et de paradoxes, celle du multiple : multiplicité des musiques abordées, certes, mais aussi multiplicité des formes d'identité.

En effet, la lecture de discours musicologiques abordant l'identité révèle rapidement que sous une même appellation se trouve une multitude de réalités qu'il est parfois difficile de relier entre elles : identité d'une œuvre face à sa nation ou à elle-même, identité d'un compositeur face au milieu ou à luimême, identité d'une nation par sa musique, identité d'un compositeur par son œuvre... Comment relier ces divers questionnements à un même mot? En quoi le concept d'identité peut-il être associé à des notions si différentes que le 
1. Peint à la lumière d'œuvres récentes (1994-2004) entendues dans le cadre de différents festivals internationaux de musique contemporaine, dont Archipel (Suisse, 2003), Ars Musica (Belgique, 2003), La $22^{\mathrm{e}}$ Biennale (Croatie, 2003), Résonance et Agora (France, 2003-2004), Musimars (Montréal, 2004). nationalisme et l'authenticité par exemple? Ou plus simplement : qu'entendon réellement par identité?

Sur le paysage global de la création musicale du tournant du siècle ${ }^{1}$, nous poserons donc un regard tout aussi global qui révèlera de multiples formes d'identité. Il fallait s'y attendre : un paysage si vaste que celui auquel nous nous attardons ne peut être embrassé d'un seul point de vue. Il sera donc question d'identité tant culturelle ou nationale que personnelle chez le créateur, tant de société que de musique, tant de conflits sans issues que de concordances évidentes.

Double multiplicité... double utopie? Peut-être. Il est impossible de donner des réponses figées et définitives à un questionnement d'ordre ontologique comme celui qui entoure l'identité; tout comme il est impossible de réellement saisir dans sa globalité la musique en devenir qu'est celle du présent. Les pages qui suivent ne prétendent donc pas donner des réponses, mais poser des questions tantôt philosophiques, tantôt sociologiques, tantôt musicologiques, voyant en ce caractère utopique un tremplin à la réflexion plutôt qu'un obstacle.

\section{Vers une définition}

Il semble incontournable de démystifier le concept d'identité avant de s'attarder à son application au monde musical actuel : quelle est sa définition, quels sont ses enjeux, ses problématiques, ses paradoxes? Sans prétendre couvrir ces questions en profondeur, les pages qui suivent visent plutôt à faire prendre conscience de l'extrême complexité du sujet. Pour ce faire, nous allons prendre pour point de départ l'utilisation usuelle du terme qui se partage en deux applications distinctes, soit l'identité individuelle et l'identité sociale.

Tout d'abord, l'identité individuelle : que signifie-t-elle au juste? Un discours très pragmatique pourrait l'associer simplement à l'identification technique d'un individu : son nom, son origine, son âge, sa taille, sa profession, bref, ses caractéristiques extérieures qui permettent de le reconnaître parmi d'autres. Bien sûr, la signification profonde du terme dépasse cette simple "carte d'identité », mais pourtant, elle relève de cette même question : «Qui suis-je? ». $\mathrm{Si}$, aux yeux du gouvernement, nous nous définissons par notre état civil, notre profession ou notre date de naissance, il est clair que, à nos propres yeux, nous sommes bien autre chose que ce simple étiquetage.

Mais qui sommes-nous? Par quoi se définit notre identité? À ces questions, aucune réponse absolue, mais un indice : nous sommes des êtres pluriels et en mouvement. Êtres pluriels, puisque nous sommes chacun composés de plusieurs facettes : nous sommes à la fois corps physique, rationnel, émotionnel, intellectuel, voire spirituel, et bien d'autres. Êtres en mouvement, puisque nous 
existons dans le temps, donc chacune de nos facettes évolue constamment, des cheveux gris (corps physique) à nos sentiments pour une personne (corps émotionnel). La recherche d'identité individuelle, liée étroitement à la recherche de notre être, est donc une quête sans fin - son objet étant mouvant - et dans laquelle nous faisons face à notre propre éclatement - notre pluralité.

Mais que recherche donc l'individu qui se questionne sur son identité? $\mathrm{Ne}$ chercherait-il pas simplement à trouver qui il est en tant que un, au-delà de sa pluralité? En ce sens, la quête d'identité ne pourrait-elle pas être liée à la recherche de l'unité, au désir de percer le mystère d'être à la fois un (en tant qu'individu) mais pluriel? Le simple fait de se poser la question suggère un désir d'avoir une réponse : la question «qui suis-je? » traduit un désir de se trouver, de se saisir, en tant que tout réuni dans un même corps. La racine latine du terme «identité » vient d'ailleurs confirmer cette hypothèse : nous y retrouvons, bien sûr, «entité», "être », mais aussi id, de idem, de «même».

Il est donc possible de supposer que la quête d'identité individuelle soit liée à la recherche d'unité ou d'harmonie chez un individu placé face à lui-même. Or, dans sa quête d'identité, celui-ci se retrouve devant une autre entité : le monde extérieur. Nous nous définissons par nous-mêmes, bien sûr, mais également en rapport avec les autres, qui exercent sur nous une influence constante, menaçant sans cesse de venir altérer cet un que nous essayons en vain de capturer. Comment y échapper, en sachant que nous ne pouvons pas nous en passer? La manière de vivre avec sans s'y confondre ne serait-elle pas d'affirmer, haut et fort, notre unicité? Non seulement je suis un, mais je suis unique : peut-être est-il impossible d'avoir la certitude de mon essence et de ma nature, mais regardez-moi, personne ne pourra venir ébranler ma conviction d'être différent de vous tous qui m'entourez. Je ne peux répondre fermement à la question "qui suis-je? ", sauf si je me contente d'y répondre que je suis moi, et non vous. Et voilà. Les autres permettent à l'individu de confirmer son existence autant qu'ils menacent de l'altérer; ils renforcent son identité en mettant en valeur son unicité. En ce sens, la quête d'identité individuelle serait à la fois, d'une part, recherche d'unité avec soi-même, et d'autre part, recherche d'unicité en rapport avec la société. Qui suis-je? Je cherche à être un, d'abord, et cet un est moi - personne d'autre.

$\mathrm{Si}$, à la base, la question identitaire relève du domaine ontologique, elle est pourtant couramment posée dans un contexte sociologique : identité nationale pour certains, appartenance à un regroupement quelconque pour d'autres. Comment comprendre cet emploi du terme identité alors qu'il est si intimement lié à l'être? Les notions d'unité et d'unicité telles qu'appliquées à l'individu proposent des pistes : l'identité sociale pourrait en effet se définir comme 
la recherche d'unité d'un individu avec un ensemble d'individus, puis la recherche d'unicité de ce regroupement par rapport à l'ensemble de la société.

Les différentes formes de quête d'identité - individuelle ou sociale - se construisent donc toutes deux sur la même dualité unité/unicité. Or, elles sont loin d'être équivalentes, bien au contraire : leur définition commune provoque une collision. Comment, en effet, être à la fois unique comme individu et uni à un groupe social quelconque? Comment être parfaitement différent et original tout en adhérant à une collectivité? La quête d'une forme d'identité impliquerait-elle nécessairement le sacrifice de l'autre?

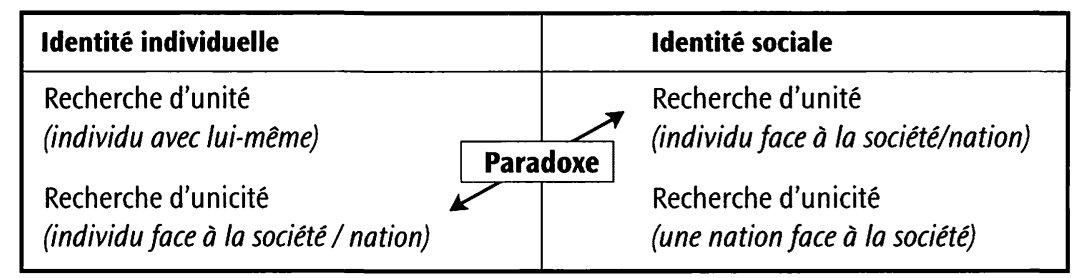

Si nous ne nous aventurerons pas dans cette réflexion pour l'instant - celleci sera possible seulement une fois l'ensemble des éléments du tableau clairement définis - nous pouvons tout de même en tirer immédiatement une leçon : la quête d'identité est en soi paradoxale. Elle place sans cesse l'un et le multiple dans des situations de combats sans issue : les deux partis doivent apprendre à coexister en harmonie sans s'anéantir. Elle éveille un besoin d'unicité chez l'individu qui, placé devant sa propre multiplicité, s'y rattache comme à une bouée de sauvetage. Nous avons déjà effleuré le sujet : cette multiplicité est inhérente à l'individu agissant comme moteur dans la recherche de sa propre unité. À quoi bon chercher mon identité si je suis clairement un? Comment prendre conscience de l'un sans prendre conscience aussi du pluriel? Comme tant de concepts, l'unité se définit par son contraire. La pluralité serait-elle à la fois l'objet dont on veut se détacher dans la quête d'identité et la raison d'être de cette même identité?

La quête d'identité met donc de l'avant l'ambiguiité du rapport entre unité et unicité, mais aussi entre l'un et le pluriel qui, tous deux, semblent se stimuler mutuellement. D'une part, la recherche d'unicité («je suis un et unique») provoque la pluralité : une société où chaque individu ou regroupement d'individus clame sa différence est forcément très diversifiée. D'autre part, la présence de pluralité provoque la recherche d'unité - chez l'individu comme dans une société précise. Or, pluralité et unité peuvent-elles réellement se rencontrer? Est-il possible de voir une pluralité comme un tout uni cohérent? 


\section{Musiques plurielles}

Cette question identitaire est particulièrement pertinente dans le domaine de la musique en ce début de millénaire, alors que même le regard le plus bref viendra confirmer sa pluralité : la musique d'aujourd'hui est d'une diversité saisissante. Contrairement à l'époque florissante de Darmstadt, on n'aspire pas à trouver la voie de l'avenir en musique : chaque compositeur semble avant tout chercher sa propre voie et non celle de son art en général. Au nom de la libre expression de chacun, tout est permis. Emprunts aux musiques populaires (Frozen Light, Derek Johnson) ou aux styles extra-occidentaux (Just Charles and Cello in the Romantic Chord, La Monte Young), retour dans le temps (Vintage, Jesper Nordin)? Mélanges disciplinaires farfelus (Happy Kitchen, Hans Joachim Roedelius), sons bruts (Confidence, Jean-François Laporte), travestissements technologiques (Strette, Hector Parra)? Musique écrite... ou improvisée (musiques actuelles)? Salle de concert «à l'italienne", lieu postindustriel (Projet Silophone, The User), paysage champêtre (Mille mètres sur terres, Rudy Decelière)? Musiciens immobiles, mobiles (Dehors / Dedans, Marie Pelletier), invisibles (Sweet Potato, Phill Niblock)? Langage néotonal, sériel, spectral, minimaliste, complexe, timbral? Construction mathématique, intuition personnelle, inspiration poétique, engagement politique? Plaisir intellectuel, sensuel, émotionnel? Comme ça vous chante : les programmations des festivals internationaux de musique contemporaine en offrent pour tous les goûts. Et ces goûts sont nombreux, tant du côté des compositeurs qui proposent chacun leur idée que de celui des auditeurs critiques qui ne tentent même pas de trouver un consensus. D'un concert à l'autre, d'un pays à l'autre, l'auditeur va de surprise en surprise : il peut s'attendre à tout... et à n'importe quoi. L'histoire a-t-elle déjà connu si grand éventail de genres, de styles, d'approches?

Cet éclatement généralisé a, bien entendu, des précédents historiques : l'éclatement de la tonalité l'annonçait déjà au début du $\mathrm{Xx}^{\mathrm{e}}$ siècle, de même que les premières recherches sur le bruit, l'apparition des musiques électroacoustiques, le renouvellement des formes de concerts avec les happenings... Ce « tout est permis» se serait donc construit petit à petit, le $\mathrm{xx}^{\mathrm{e}}$ siècle ayant ouvert une à une les portes de chacun des paramètres musicaux, les libérant de leurs contraintes. Et aujourd'hui? Le XXI ${ }^{\mathrm{e}}$ siècle naissant récolterait le fruit de ces efforts : toutes les portes semblent ouvertes. Le compositeur n'a plus de limites, plus de barrières à contourner ni à bousculer : il est libre.

Parallèlement à cette pluralité externe de l'ensemble du paysage musical actuel précédemment défini, un regard un peu plus attentif saura noter une pluralité interne, c'est-à-dire intrinsèque à l'œuvre musicale en elle-même. Il peut paraître étrange de se prononcer sur l'œuvre musicale au singulier dans 
3. Comme l'auteur l'expose plus en détails dans le cinquième volume de "Musiques : une encyclopédie pour le XXIe siècle" (Pascal, N., à paraître). cet univers de pluralité : l'immense diversité - non seulement observée, mais aussi revendiquée - laisse croire en l'impossibilité de tout discours globalisant. Toutefois, en demeurant conscient des différences et des exceptions, quelques traits sont particulièrement frappants par leur récurrence ou par leur intensité, ceux-ci tournant autour du concept même de diversité.

Cette diversité se traduit notamment par l'abondance de «mélanges» : les compositeurs n'hésitent pas à fusionner dans une même œuvre des éléments de prime abord très disparates. Par exemple, la pluridisciplinarité est extrêmement présente sur la scène musicale, allant bien au-delà de la traditionnelle fusion musique/théâtre au sein de l'opéra. Musique et arts visuels dans le cas des installations et sculptures sonores ou encore, très fréquemment, dans le cas des œuvres alliant musique et vidéo; musique et danse, bien sûr, sous des formes très diversifiées; musique et théâtre, de l'opéra traditionnel au théâtre musical ou instrumental; et, dans certains cas isolés, musique et art culinaire (Happy Kitchen, Hans Joachim Roedelius) ou mode vestimentaire (Musique défilé pour une fin de siècle, Marcelle Deschênes et Linda Bouchard). Évidemment, de semblables mélanges ont marqué l'ensemble de l'histoire - chacun des genres puise ses racines dans des époques passées - mais ils semblent connaître aujourd'hui une ouverture sans pareille, aidés notamment par la technologie elle-même très large. En effet, les nouveaux moyens techniques permettent la réalisation de concepts autrefois irréalisables : un danseur contrôlant, par ses mouvements, des sons émis par un musicien (Connected Spaces, Alastair Bannerman); un chanteur devenant acteur lorsqu'on le met en scène avec, entre les mains, un ordinateur portable traitant sa voix en temps réel (Dichterlesung, Thomas Kessler). Bref, tous les mélanges disciplinaires sont permis, mais aussi tous les mélanges disciplinaires sont possibles, sous toutes les formes d'interaction imaginables.

Outre les mélanges disciplinaires : des mélanges d'outils. Alors que pendant longtemps une barrière séparait le monde des musiques acoustiques et électroacoustiques, ces deux univers se croisent maintenant dans un genre dont le nom suggère en lui-même une forme de pluralité : les musiques mixtes. Cellesci semblent prendre une place croissante sur la scène musicale contemporaine, animées d'un nouvel élan grâce aux techniques permettant le traitement électronique du son en temps réel - techniques auxquelles se consacrent de nombreuses institutions dont l'Ircam. Aujourd'hui, l'œuvre n'est plus nécessairement instrumentale ou électroacoustique : elle peut être les deux à la fois.

Une pluralité semblable se retrouve dans l'orientation stylistique en soi de beaucoup d'œuvres, qui n'hésitent pas à mélanger musiques «savante » et "populaire», musiques d'ici et d'ailleurs, musiques de telle école ou de telle autre. Gestes populaires, notamment par l'élan rythmique de certaines œuvres 
(Go, James Sellars); gestes d'ailleurs, par l'emprunt de modes particuliers (Just Charles and Cello in the Romantic Chord, La Monte Young) ou, encore une fois, d'instruments exotiques (Gatekeepers, Tomi Raisanen); gestes d'écoles a priori distinctes lorsqu'un compositeur intègre, par exemple, des techniques spectrales dans un discours très loin du spectralisme (Barocco, Mauro Lanza). Bref, les œuvres d'aujourd'hui se donnent la liberté d'aller puiser à gauche et à droite, mélangeant les influences sans nécessairement se soucier de s'attacher à une tendance particulière.

Bien sûr, ces types de mélanges - entre disciplines, genres ou esthétiques ne marquent pas nécessairement toutes les œuvres d'aujourd'hui, comme ils ne sont pas nécessairement uniques à notre époque : un bref regard sur l'histoire de la musique occidentale permettrait d'en repérer des traces même dans les époques les plus lointaines, mais leur importance n'en est pas moins grande. Aujourd'hui, ils se dressent fermement de part et d'autre du milieu musical, encouragés même par les institutions les plus puristes de réputation. Par exemple, l'Ircam (Paris), destiné originairement à la recherche en musique, consacre annuellement depuis 1998 un festival (Agora) voué à la promotion des rapports entre musique et autres formes artistiques. Certains ensembles instrumentaux intègrent eux-mêmes à leur programme des œuvres «plus-qu'instrumentales " : le London Sinfonietta, par exemple, mettait à son programme lors d'un concert à Zagreb deux œuvres de musique électroacoustique accompagnées de vidéo. De même, le Nouvel Ensemble Moderne (Montréal), voué au départ à la musique instrumentale, mettait sur pieds en 2002 la première tribune canadienne de musique mixte avec MusMix. Pour ce faire, il s'associait à un organisme qui se consacrait jusqu'alors à la musique acousmatique, Réseaux - qui, d'ailleurs, annonçait deux ans plus tard la dernière édition de son festival «Rien à voir »... qui sait, peut-être pour se renouveler dans un futur "Quelque chose à voir »?

La liste d'exemples pourrait être longue, et les débats autour de tels choix aussi : qu'est-ce qui incite les organisateurs d'événements, et même les compositeurs et interprètes, à s'ouvrir à de tels «mélanges »? Certains parleraient de tendance esthétique, simplement, alors que d'autres accuseraient un désir - politique et économique - d'atteindre un public plus large. Car en effet, ces «mélanges» trahissent souvent un grand souci de perceptibilité : le plaisir musical physique n'est plus snobé comme au temps de la modernité, et même au contraire, il est valorisé, incarné par l'emploi de sonorités sensuelles (Ondulado tiempo sonoro, Alberto Posadas), de rythmes envoûtants (Go, James Sellars) ou de dynamiques extrêmes (Transmission, Franck Bedrossian), toujours au service de l'expressivité. 
4. Scarpetta, G. (1985), L'impureté, Paris, Grasset. L'auteur définit "l'esthétique de l'impureté" comme étant l'esthétique du mélange.
Il serait bien sûr possible de mettre cette «impureté4 » assumée sur le dos du "postmodernisme» : nous n'entrerons pas dans cette discussion, qui risque de prendre l'allure d'une guerre terminologique. Peu importe son nom, son ascendance et même ses causes, une pluralité certaine habite la musique d'aujourd'hui, tant sur le plan «interne» de l'œuvre par les mélanges qu'elle met en scène que sur le plan «externe» par la diversité du paysage musical dans son ensemble.

Quelles en sont les conséquences? Parmi tant d'autres, probablement, une impression de confusion sur plusieurs plans. D'une part, confusion des genres et des styles : à force de mélanges, toute catégorisation devient futile. Il n'y a plus de limites, plus de frontières d'un style à l'autre ou d'un genre à l'autre, mais plutôt des «zones grises» qui appartiennent à la fois à toutes les catégories et à aucune, des «zones grises » qui, par leur ampleur, en viennent à faire oublier les «zones claires». D'autre part, confusion des rôles : les compositeurs deviennent tantôt sculpteurs (dans les installations sonores), metteurs en scène (du côté du théâtre musical), vidéastes (dans les œuvres de «vidéomusique ») ou interprètes performeurs; les interprètes quant à eux deviennent tantôt acteurs (encore une fois, dans le théâtre musical) tantôt compositeurs (dans les œuvres qui laissent place à l'improvisation), et même les auditeurs deviennent parfois interprètes (dans certaines œuvres interactives). Bref, plus aucune fonction n'est figée, définitive : les barrières tombent entre les différents intervenants de l'œuvre musicale semant sans contredit une certaine confusion.

\section{Grandeur et misère de l'identité musicale dans un monde de pluralité}

Comment s'y retrouver dans cet univers musical où la pluralité extrême crée parfois un véritable brouillard? Comment saisir l'identité de l'œuvre, de son auteur, de son auditeur, alors que ce qu'on leur croyait inhérent est ébranlé? Reposons la question déjà mentionnée : est-il possible de voir une pluralité - cette foisci en musique - comme un tout uni cohérent? La pluralité propre à la musique d'aujourd'hui sème la confusion autour de l'un et de l'unique tant du côté de l'œuvre que de celui de son créateur et de son auditeur : la question «qui suisje?» semble y prendre un caractère plus incertain que jamais. Car même les caractéristiques «extérieures", qui ne touchent pas à son essence mais simplement à sa description technique, deviennent confuses.

Premier défi à relever, dans ce monde de pluralité, pour se rapprocher de son identité : trouver son unité au-delà de la diversité. D'abord, le cœur du phénomène musical, l'œuvre en elle-même. Qu'en est-il de son identité alors que, aujourd'hui, elle se définit par une esthétique interne de la pluralité? L'œuvre d'esthétique impure doit faire face, plus que tout autre probablement, à la 
problématique de l'un : parvenir à se présenter en tant que tout cohérent indivisible malgré la variété d'outils, de gestes, de styles ou de sensations mise en scène. Ensuite, le créateur de cette œuvre : comment peut-il trouver son identité en tant qu'individu à travers son art alors que son art lui-même est aussi pluriel et insaisissable que sa propre personne? Comment peut-il se définir alors que la mixité de son œuvre - et de son milieu - rend toute catégorisation impossible? Pour dévoiler son identité, le compositeur d'aujourd'hui ne peut pas simplement s'identifier à une école ou à un courant précis : ceux-ci tendent à perdre leur signification par l'apparition de «zones grises». Le compositeur doit donc démontrer sa propre unité, si insaisissable soit-elle, sans pouvoir se rattacher aux balises dressées par le milieu musical. Et l'auditeur? Comment, à son tour, peut-il saisir l'œuvre alors que règne autour d'elle la complexité du multiple? Il doit faire preuve d'une immense ouverture et d'une capacité d'adaptation hors du commun, certes, mais encore : nous pourrions soupçonner chez lui l'importance de préserver sa propre identité face aux nombreuses œuvres spectaculaires qui lui imposent un univers perceptif donné. À plus grande échelle, comment une nation peut-elle trouver son unité culturelle avec, en mains, des œuvres qui se veulent uniques dans leur pluralité? À ces défis d'unité posés à toutes les sphères de la musique d'aujourd'hui s'ajoute le défi d'unicité, de loin plus facile à saisir en théorie mais tout aussi problématique dans le contexte actuel : que signifie l'originalité d'une œuvre ou d'un compositeur si l'originalité est la marque de toutes les œuvres de leur époque?

À cette étape, la complexité de la question identitaire en musique aujourd'hui paraît évidente, liée très étroitement à la pluralité qui la caractérise. Mais comment se sortir d'un tel labyrinthe? Au tournant du siècle, l'individu face à la musique de son temps - le compositeur comme l'auditeur - est-il dans une impasse? Doit-il se résigner à oublier la possibilité de l'un et unique et accepter, à tout prix, la présence du pluriel au risque de perdre ses repères et de sombrer dans la confusion? Bien entendu, impossible de répondre avec certitude à ces questions. Toutefois, en relevant quelques-uns des pièges du monde de la pluralité ${ }^{5}$, il est possible d'imaginer, par contraste, les moyens d'atteindre la solidité de l'un - bref, d'une identité.

Le risque le plus évident dans un univers musical de confusion est celui d'un manque d'harmonie. En effet, en prenant la liberté de mettre ensemble des éléments disparates dans leur œuvre - que ce soit d'un point de vue stylistique, instrumental ou autre - les compositeurs courent le risque de créer une œuvre «dissonante ». Combien de fois, par exemple, l'oreille est-elle choquée par une fusion maladroite entre des sons électroniques et acoustiques dans des œuvres mixtes? Par la domination de l'image sur le son dans des
5. Qui se rapprochent étroitement des pièges de l'esthétique de l'impureté, comme l'a développé l'auteur dans le cinquième volume de "Musiques: une encyclopédie pour le XXıe siècle" (Pascal, N., à paraître). 
œuvres pluridisciplinaires? Dans une esthétique plus puriste, le problème de l'harmonie n'est-il pas assurément moins frappant (sans vouloir dire qu'il soit absent)? Bien sûr, par définition la musique est «mélange ». Bien sûr, la question de l'harmonie et de l'unité est inhérente au phénomène musical, mais face à un monde de pluralité, celle-ci prend une allure beaucoup plus flamboyante. La dissonance bien dosée peut certainement avoir quelque chose de séduisant à l'oreille, qui aime bien être dérangée, mais elle se confond parfois avec la notion de déséquilibre et l'œuvre, à partir de ce moment, ne peut que difficilement se faire voir comme un tout à l'identité bien définie.

Deuxièmement, parallèlement à ce risque de dissonance, l'univers de la pluralité offre des pièges d'uniformité. Étrange? L'harmonie est un funambule qui risque à tout instant de tomber du côté de la dissonance grinçante ou de celui de l'uniformité ennuyeuse; et ces deux côtés, si opposés soient-ils, se rejoignent sur le même plan. À force de différences, la différence perd de son impact; à force de diversité, d'unicité, d'originalité de toutes parts, plutôt que d'une mise en valeur de l'identité, une impression d'uniformité. À l'intérieur de l'œuvre : l'instrument le plus exotique aux sonorités les plus particulières pourra-t-il être apprécié dans toute son unicité s'il est mêlé à une instrumentation spectaculaire réunissant des instruments acoustiques, électroniques, une trame électroacoustique, et le tout, pourquoi pas, accompagné d'une vidéo (East Aka West, Ricardo Nova)? Il semble y avoir un risque de «contagion» lorsque divers éléments partagent le même espace : tout peut facilement devenir tout, chaque élément perdant une partie de sa spécificité, créant finalement, au-delà des différences, une certaine uniformité. La particularité d'une unité se noie facilement dans le commun de la pluralité...

Mais laissons ici de côté l'œuvre elle-même, déjà bien occupée à éviter les dissonances, pour observer le milieu musical actuel dans son ensemble. Que se passe-t-il? Il semble extrêmement difficile pour les compositeurs de se faire remarquer : puisqu'il est acquis que tout est permis et possible, comme nous l'exposions plus haut, plus rien n'étonne. Certains prennent part à une «course à l'originalité» espérant toujours qu'en battant le record, leur unicité sera reconnue; d'autres au contraire baissent les bras devant l'ampleur du défi. Par chance, ces deux attitudes - l'originalité pour l'originalité autant que la désillusion face à l'unicité - n'habitent apparemment pas tous les compositeurs d'aujourd'hui, mais le risque semble bel et bien présent, témoin d'un malaise face à la perte de signification de la différence.

Les «zones grises» déjà évoquées en sont certainement en partie responsables : le contraste entre deux styles ou deux genres n'est-il pas amoindri lorsqu'ils partagent un point de rencontre? Par la liberté des mélanges, de multiples 
dégradés servent de liens entre des éléments a priori opposés. C'est ainsi que la diversité de l'ensemble de la musique d'aujourd'hui peut parfois donner l'impression d'une unique masse multicolore, les différentes écoles de musique de tradition classique se confondant entre elles, puis avec les musiques du monde ou populaires. Le phénomène semble même venir ébranler ce qu'on pourrait appeler les «écoles nationales» : des professeurs de toutes origines enseignent partout à des étudiants compositeurs de toutes origines. Il y a donc, encore une fois, une tendance à l'uniformisation. Et à force d'uniformité, alors que «tout peut être tout ", semant la confusion, il risque d'être très difficile de saisir l'un.

À ces deux pièges de l'univers de la pluralité - dissonance ou uniformité s'en ajoute un troisième, à la fois cause et conséquence des deux précédents : le risque de perdre tout jugement ou d'accepter tout aveuglément, même le «kitsch", au nom de la «libre expression » d'un individu. Le reconnu saxophoniste français Claude Delangle enchaînant sans interruption du Reich et du Berio parmi d'autres œuvres sur une chorégraphie anecdotique à la limite du vulgaire (Zagreb, 11 avril 2003)? Pourquoi pas! Face à l'extrême diversité qui les entoure, l'auditeur et le compositeur se trouvent facilement démunis : quelle direction emprunter parmi toutes? La solution la plus simple est certainement de les emprunter toutes. Or, accepter «n'importe quoi » sans faire intervenir son propre jugement n'aide certainement pas à se rapprocher de son identité. Au contraire, l'acceptation aveugle ne serait-elle pas plutôt un signe de faiblesse identitaire, d'envahissement de l'un par les autres?

Une fois ces quelques pièges découverts, imaginons des moyens de les éviter pour favoriser un accès à la découverte et à l'expression de l'un. Pour éviter la dissonance ou l'uniformité? Viser l'harmonie, l'équilibre, la mesure, même dans les débordements. Pour éviter l'avilissement par la mort de notre propre jugement? S'assurer d'être actif, lucide, ne jamais cesser de se questionner. Trouver l'identité d'une œuvre, d'un compositeur, d'une nation, serait-il si simple? Malheureusement - ou heureusement - les quelques solutions évoquées ici sont des utopies : personne ne pourrait se vanter d'avoir atteint l'harmonie parfaite, ni jurer de ne jamais céder à l'appel du moindre effort. La quête d'identité - d'unité et unicité - est sans fin : ne serait-elle pas alors un moteur à la création dans notre monde de pluralité?

Nous pourrions donc croire que si la pluralité en musique aujourd'hui est la cause de bien des maux et des angoisses, elle est aussi responsable de sa grandeur. Car une fois les "pièges " évités, le monde musical actuel n'est-il pas extrêmement stimulant? Sa diversité, pour ceux qui ne sombrent pas dans la passivité, ne provoque-t-elle pas sans cesse l'exploration, la recherche, les réflexions, pour aider à mieux se définir? L'ouverture à la différence est une 
6. Comme le souligne E. J. Hobsbawm ([1999], L'âge des extrêmes. Histoire du court $X x^{e}$ siècle, Éditions Complexe, p. 36), "le monde s'est modifié en une unité opérationnelle unique".

7. Molino, J. (2003), "Technologie, mondialisation, tribalisation ", dans Musiques: une encyclopédie pour le XXI" siècle, tome I, Arles, Actes Sud.

8. Taylor, C. (1992), Grandeur et misère de la modernité, Québec, Bellarmin. richesse, préparant le terrain à l'éclosion d'idées et d'œuvres autrement latentes. La pluralité serait donc elle aussi bel et bien une richesse, dans la mesure où on ne perd pas de vue l'idéal de l'unité.

\section{Identité sociale/identité musicale : un rapport de causalité ?}

L'identité de la musique d'aujourd'hui, dans son ensemble, est donc étroitement liée au concept de pluralité. Cette tendance pourrait certainement s'expliquer par l'axe chronologique de la modernité/postmodernité, mais une seconde voie semble plus à propos dans le cadre de cette étude : l'identité musicale de notre époque ne pourrait-elle pas être liée à son identité sociale? Comme nous le mentionnions en début de parcours, l'identité est un concept aux multiples facettes, lié à l'unité d'un tout face à lui-même, certes, mais également à l'unité d'un tout face à la réalité qui l'entoure. Oui, les musiques étudiées ici ont une identité de pluralité, mais que sont-elles en rapport avec le monde dans lequel elles évoluent? En quoi sont-elles unies à leur environnement social?

Un regard sur la société actuelle, même très bref, permettrait d'établir quelques équivalences, liant chacune des caractéristiques musicales relevée à une caractéristique sociale. Quelles sont ces caractéristiques sociales? Sans entrer dans les détails, il apparaît évident que la société du tournant du siècle est une société de pluralité, où les frontières et contraintes géographiques, hiérarchiques, voire morales ou techniques tendent à s'estomper, provoquant une multiplicité illimitée, généralisée, elle-même source d'une sensation d'uniformitét. Plus précisément, le Xxi ${ }^{\mathrm{e}}$ siècle naissant, comme le souligne Molino, serait un siècle de «mondialisation », de «tribalisation » et de «technologie" »; ou encore, selon Taylor, de "despotisme», de "raison instrumentale ${ }^{8}$ » et « d'individualisme ${ }^{9}$ » - tant de notions qui se lient d'elles-mêmes aux caractéristiques musicales définies dans le tableau ci-contre (p. 67).

Le « tout est permis » propre à la musique de la décennie étudiée est donc en union avec un «tout est permis» social généralisé, que Taylor explique ainsi :

Chacun a le droit d'organiser sa propre vie en fonction de ce qu'il juge vraiment important et valable. Il faut être sincère envers soi-même et chercher en soi-même son propre épanouissement. En quoi consiste cet épanouissement? En dernière analyse, c'est à chacun de le déterminer pour soi-même. Personne d'autre ne peut ou ne doit essayer de lui dicter quoi que ce soit ${ }^{10}$.

Comme nous le soulignions au sujet des tendances musicales, cette grande tendance sociale n'est pas de "génération spontanée » et dépourvue d'antécédents. Au contraire, l'idéal de la liberté individuelle remonte à plusieurs décennies, dont le nombre varie d'une culture à l'autre. Or, à nos yeux de contemporains, elle semble plus frappante que jamais; et à nos yeux de 


\begin{tabular}{|l|l|}
\hline \multicolumn{1}{|c|}{ Caractéristiques musicales } & \multicolumn{1}{c|}{ Caractéristiques sociales } \\
\hline $\begin{array}{l}\text { Relativisme face au jugement esthétique } \\
\text { (tout est permis) }\end{array}$ & $\begin{array}{l}\text { Relativisme face au jugement de valeurs } \\
\text { (tout est permis) }\end{array}$ \\
\hline $\begin{array}{l}\text { Importance de la "libre expression" } \\
\text { du compositeur } \\
\text { Importance du plaisir de l'auditeur }\end{array}$ & $\begin{array}{l}\text { Individualisme } \\
\text { ("Idéologie de l'épanouissement de soi" ") }\end{array}$ \\
\hline $\begin{array}{l}\text { Intégration d'éléments de musiques } \\
\text { d'ailleurs }\end{array}$ & Mondialisation \\
\hline $\begin{array}{l}\text { Intégration d'éléments de musiques } \\
\text { "populaires" }\end{array}$ & "Tribalisation" \\
\hline $\begin{array}{l}\text { Les nouvelles technologies à l'assaut } \\
\text { de la musique }\end{array}$ & $\begin{array}{l}\text { Valorisation de la technologie/ } \\
\text { Importance du progrès }\end{array}$ \\
\hline $\begin{array}{l}\text { Importance du plaisir de l'auditeur } \\
\text { Importance du "spectacle" }\end{array}$ & $\begin{array}{l}\text { Système capitaliste basé sur la production } \\
\text { et la consommation } \\
\text { Importance de l'" efficacité" }\end{array}$ \\
\hline Diversité extrême ayant pour conséquence une tendance à l'uniformisation \\
\hline
\end{tabular}

chercheurs, cette impression se confirme, du point de vue social comme il en a été du point de vue musical : les générations précédentes ont ouvert graduellement des portes, au point d'en arriver aujourd'hui à ne plus en avoir aucune qui soit fermée.

\section{Identités nationales}

Les caractéristiques musicales et sociales posées ci-dessus, en regroupant plusieurs musiques et plusieurs nations, font abstraction d'une forme d'identité bien présente dans les discours de certains compositeurs et musicologues d'aujourd'hui : l'identité nationale. Si ce sujet est bel et bien d'actualité, les réactions qu'il suscite sont pourtant loin d'être unidirectionnelles : hormis ceux qui en sont totalement détachés (car il y en a), plusieurs tendances se laissent voir dans les rapports qu'entretiennent les compositeurs avec leur nation ${ }^{12}$, que nous pourrions identifier ainsi :

1. Nationalisme revendiqué

2. Nationalisme d'adoption

3. Mondialisme

4. Nationalisme acquis

Le «nationalisme revendiqué» est certainement le plus apparent, simplement parce que ses adeptes tiennent à se faire entendre et voir comme tels.
11. Taylor, C., ibid.

12. Toujours à la lumière d'œuvres récentes (1994-2004) entendues dans des festivals internationaux de musique contemporaine en Europe et au Canada. 
13. Propos recueillis par Simon Bertrand lors d'une entrevue à la Faculté de Musique de l'Université de Montréal, le 3 novembre 2004.
Au Québec, des Denys Bouliane ou Yannick Plamondon défendent ouvertement une musique québécoise qui se démarque de ses cousines américaines ou européennes. De même en Croatie, alors que le discours de plusieurs compositeurs laisse voir une préoccupation nationaliste et que certaines œuvres en sont marquées de manière évidente - comme c'est le cas par exemple de l'opéra Animal Farm de Igor Kuljeric, dont la thématique est étroitement liée à la réalité politique du pays.

$\mathrm{Ce}$ «nationalisme revendiqué » pourrait sembler être une réaction à deux autres attitudes actuelles en musique aujourd'hui : le «nationalisme d'adoption» et le «mondialisme». La première expression est employée ici pour désigner la tendance de certains compositeurs à adopter, consciemment ou non, un style propre à une nation qui n'est pas la leur : combien de compositeurs étrangers basés à Paris ont une approche musicale plus française que les Français eux-mêmes? Ou combien de compositeurs non américains s'identifient - plus ou moins consciemment - à l'école américaine? Comme le soulignait le compositeur brésilien Guilherme Carvalho lors d'un entretien public donné dans le cadre du septième Forum du Nouvel Ensemble Moderne $^{13}$, il peut être tentant pour un compositeur originaire d'une nation dont la tradition musicale classique (occidentale) est moins affirmée de s'attacher à une école extrêmement bien établie comme le sont les écoles française et américaine. Outil pour assurer une certaine crédibilité à son travail, témoignage d'une fascination pour les nations berceaux de la tradition musicale classique ou résultat logique de l'émigration des intellectuels vers les grands centres? Peu importe, le «nationalisme d'adoption» est une réalité bien présente aujourd'hui, tout comme l'est le «mondialisme » ci-haut cité, qui désigne quant à lui cette attitude très courante chez les compositeurs de piger leur inspiration à gauche et à droite, dans la tradition musicale de divers pays. Toujours dans le cadre du Forum du NEM, le compositeur Ondrej Adamek (République tchèque) nous a donné un bon exemple de ce «mondialisme » avec Sinuous Words, œuvre inspirée tant de chants traditionnels du pays d'origine du compositeur que de chants provenant de la Nouvelle-Calédonie.

Aux côtés de ces compositeurs engagés dans une recherche nationaliste ou mondialiste, d'autres, sans être engagés du tout, proposent une musique malgré tout clairement attachée à leur nation : ils sont habités d'un «nationalisme acquis ». Reprenons le cas classique de l'école française : plusieurs œuvres demeurent empreintes de l'esprit français sans pour autant le revendiquer. Duo Concertante de Laurent Torres par exemple : oui, nous y retrouvons des traits évidents d'identité nationale, non pas en guise de quête ou de preuve, mais plutôt comme acquis. "Ça va de soi », tout simplement. 


\section{Identitê nationale et individuelle : éviter la collision}

Les quatre catégories esquissées ici pour illustrer les différents visages du nationalisme musical d'aujourd'hui mériteraient certainement d'être approfondies davantage, mais tel n'est pas l'objectif de la présente étude : nous aspirons plutôt à élucider la problématique de l'identité musicale dans le monde de pluralité qui est le nôtre, et, comme nous l'avons vu, celle-ci comporte plusieurs facettes. D'une part, l'identité individuelle : celle de l'œuvre, de son créateur et de son auditeur; d'autre part, l'identité sociale globale qui se partage elle-même en de multiples identités nationales. Comme nous l'avons observé dans le graphique de la page 58 , certaines de ces identités entrent en collision. Maintenant que nous avons défini chacun des éléments du tableau, attardons-nous plus particulièrement à cette friction qui, de prime abord, nous place devant une impasse.

Quel était donc l'enjeu de la collision? Résumons ici la dynamique de la quête d'identité nationale - à laquelle seuls le «nationalisme revendiqué » et le «nationalisme adopté » se rattachent, le «nationalisme acquis» et le «mondialisme» n'étant quant à eux pas associés à une quête - en reprenant les principes directeurs tirés de notre réflexion sur l'identité de l'œuvre musicale. Premièrement, rappelons-le, viser l'harmonie, puis deuxièmement, ne pas se laisser tenter par la passivité : selon ces critères, la quête identitaire nationale en musique trouverait son salut dans l'activisme et dans la recherche de cohésion avec les autres. Or, face à cette réponse, un certain malaise persiste : une telle quête d'identité nationale ne se ferait-elle pas nécessairement au détriment de l'identité de l'individu, plus précisément de l'artiste et de son œuvre? La quête de l'identité sociale peut éventuellement impliquer le sacrifice de l'identité individuelle : ici se trouve la collision. L'artiste devrait-il alors forcément faire un choix entre son identité personnelle et son identité sociale?

Prenons alors une chance : parmi les deux voies qui s'offrent à nous, optons pour celle de l'identité personnelle et imaginons la suite. Si chaque artiste, chaque compositeur, se préoccupait avant tout de se définir lui-même par son œuvre tout en s'assurant que cette œuvre soit bel et bien une en elle-même, quel serait le résultat d'un point de vue social ou national? La «nation » auraitelle une cohérence à son tour, une unité et une unicité, ou se perdrait-elle dans l'uniformité universelle? Si nous nous fions à notre détour sociologique, la relation société/musique est une relation - partiellement - de cause à effet : l'identité musicale nationale n'irait-elle donc pas de soi, émanant naturellement de tout peuple? Et si nous avions emprunté l'autre chemin, celui de l'identité sociale : pourrions-nous supposer que la quête d'identité nationale en musique puisse à son tour provoquer l'identité personnelle de l'œuvre et de son auteur dans un même rapport de causalité? Malheureusement, l'équation n'est pas 
valable en ce sens : il ne suffit pas à l'artiste de s'identifier à sa nation pour découvrir sa propre identité. Mais consolons-nous, ce que nous croyions être une impasse n'en est peut-être pas une : la première voie empruntée, celle de l'identité personnelle en art, peut déboucher tout naturellement sur la seconde voie, celle de l'identité nationale, sans que nous n'ayons eu à la chercher. Pour valoriser l'identité nationale en art, peut-être suffirait-il alors simplement de lui faire confiance et de rendre à César ce qui lui appartient - remettre l'activisme entre les mains des politiques et charger les artistes de manifester leur identité individuelle la plus profonde.

La quête d'identité nationale, une question de confiance? Nous parlions en début d'article de la quête d'unicité comme d'une bouée de secours face à l'inatteignable unité. Or, qui a besoin d'une bouée : le fort ou le vulnérable? Le confiant ou le complexé? Une chose est frappante sur la scène musicale internationale : le questionnement identitaire national actif ( «nationalisme revendiqué» ou "nationalisme d'adoption») semble marquer plus particulièrement les «petites» ou «jeunes» nations, désireuses de «prouver» leur unicité. Par exemple, Zagreb, Croatie, mars 2003 : à la sortie de la création d'un opéra du compositeur «national» Igor Kuljeric, Animal Farm, le regard d'une jeune journaliste s'émeut en entendant un auditeur étranger souligner la particularité stylistique de l'œuvre et clamer qu'on n'aurait certainement jamais entendu chose semblable dans son pays d'origine. Que reflète ce regard ému? Une certaine fierté, bien sûr, mais aussi, probablement, un soupir de soulagement : l'unicité de sa nation est confirmée en venant, par le fait même, justifier sa raison d'être. Pouvons-nous imaginer semblable scénario en France, par exemple? Pouvonsnous imaginer les yeux d'un journaliste français briller avec autant d'éclat au simple énoncé du «style français »? Deux extrêmes ici : la nation fragilisée en manque de confiance, puis la nation solide, forte, au «nationalisme acquis »... peut-être parfois en manque d'humilité. Chacune comporte ses richesses, l'une fraîche et émerveillée, l'autre solide et inébranlable, mais chacune comporte aussi ses risques et ses pièges, l'une au bord d'un activisme faisant mauvais ménage avec la musique, l'autre au bord d'une complaisance passive. Car audessus de toute quête d'identité nationale continuent à planer les mots d'ordre de l'identité individuelle - de l'œuvre ou de son auteur : harmonie et jugement.

\section{La victoire de l'un au-delà du multiple}

Notre parcours, partant d'une définition de l'identité autour des concepts d'unité et d'unicité, n'a certainement pas permis de répondre solidement à toutes les interrogations musicologiques, philosophiques, sociologiques. Il a toutefois ouvert quelques portes à la compréhension des identités musicales 
d'aujourd'hui, reliant entre eux deux angles d'approche distincts, ontologique et sociologique, autour de la problématique de l'un à travers le multiple.

Problématique, vraiment? Oui, lorsque nous pensons à la difficulté de l'individu compositeur de trouver sa place dans ce monde d'extrême diversité; oui, lorsque nous pensons à la difficulté, pour une œuvre «d'esthétique du mélange ", de trouver harmonie et équilibre; oui, lorsque nous pensons à la difficulté d'une nation de se distinguer culturellement dans un monde où l'absence de frontières et de limites tend à provoquer une uniformité généralisée. Problématique, sûrement, mais pourtant source de richesses lorsqu'on ne perd pas de vue l'idéal de l'harmonie et l'importance du jugement - moral ou esthétique : la multiplicité stimule la recherche de l'un et, par le fait même, contribue à sa construction. Peut-être pourrions-nous ainsi supposer que plus la pluralité est grande, plus l'unité qui parvient à s'en dégager peut être forte? Qui sait : l'identité - musicale, individuelle, sociale, nationale - dans ce monde de pluralité connaît peut-être à la fois ses moments les plus difficiles mais aussi, si elle en sort vainqueur, ses moments les plus grands...

\section{BIBLIOGRAPHIE}

Agora, programme de festival, Paris, 2-16 juin 2004 .

Archipel, programme de festival, Genève, 30 mars-6 avril 2003.

Ars musica, programme de festival, Bruxelles, 6-23 mars 2003.

BARICCO, A. (1998), L'âme de Hegel et les vaches du Wisconsin, Paris, A. Michel.

Bosseur, J.-Y. (1992), Vocabulaire de la musique contemporaine, Paris, Minerve.

Charles, D. (2001), Fiction de la postmodernité selon l'esprit de la musique, Paris, Presses Universitaires de France.

Compagnon, A. (1990), Les cinq paradoxes de la modernité, Paris, Seuil.

Hobsbawn, E. J. (1999), L'áge des extrêmes. Histoire du court XX' siècle, Éditions Complexe.

JaNkElevitch, V. (1960), Le pur et l'impur, Paris, Flammarion.

Moles, A. (1971), Psychologie du kitsch : l'art du bonheur, Paris, Denoël/Gonthier.

Molino, J. (2003), “Technologie, mondialisation, tribalisation », dans Musiques : une encyclopédie pour le XXJ' siècle, tome 1 : musique du XX'e siècle, s. la dir. de Nattiez, J.-J., Arles, Actes Sud, p. $69-88$.

Musimars, programme de festival, Montréal, SMCQ / faculté de musique de l'université McGill, $1^{\mathrm{er}}-6$ mars 2004.

Pascal, N., "Musiques au tournant du xxe siècle : l'unité dans la diversité », dans Musiques : une encyclopédie pour le XXI' siècle, tome 5, s. la dir. de Nattiez, J.-J., Arles, Actes Sud, à paraître.

Ramaut-Chevassus, B. (1998), Musique et Postmodernité, Paris, Presses Universitaires de France.

Résonances, programmes de festival, Paris, Ircam, 15-24 octobre 2003.

Rien à voir (15), programme de festival, Paris, Réseaux, 11-15 février 2004.

Scarpetta, G. (1988), L'artifice, Paris, Grasset.

Scarpetta, G. (1985), L'impureté, Paris, Grasset.

TAYLOR, C. (1992), Grandeur et misère de la modernité, Québec, Bellarmin.

${ }_{22}{ }^{\text {nd }}$ Music Biennale Zagreb - intermational festival of contemporary music, programme de festival, Zagreb, 4-12 avril 2003. 


\begin{tabular}{|c|c|c|c|}
\hline Compositeurs & Cuvres & Genre/instrumentation & Lieux de diffusion/diffuseurs \\
\hline $\begin{array}{l}\text { Adamek, Ondrej (Rép. } \\
\text { tchèque, 1979) }\end{array}$ & Sinuous Words (2004) & Ensemble instrumental & $\begin{array}{l}\text { Montréal, NEM. Novembre } \\
2004 .\end{array}$ \\
\hline $\begin{array}{l}\text { Bannerman, Alastair } \\
\text { (Angleterre, 1972) }\end{array}$ & Connected Spaces (2003) & $\begin{array}{l}\text { Flûte, danse et traitement } \\
\text { électronique en temps réel }\end{array}$ & Paris, Ircam. Octobre 2003. \\
\hline $\begin{array}{l}\text { Bedrossian, Franck } \\
\text { (France, 1971) }\end{array}$ & Transmission (2002) & Basson et électronique & Paris, Ircam. Juin 2004. \\
\hline $\begin{array}{l}\text { Deschênes, Marcelle } \\
\text { Bouchard, Linda } \\
\text { (Canada, 1939,1957) }\end{array}$ & $\begin{array}{l}\text { Musique. Défilé pour } \\
\text { une fin de siècle (2000) }\end{array}$ & $\begin{array}{l}\text { Ensemble instrumental, } \\
\text { électronique, défilé de mode }\end{array}$ & Montréal, NEM. Février 2000. \\
\hline Decelière, Rudy (Suisse, 1979) & Mille mètres sur terre (2003) & Installation sonore in situ & Genève, Archipel. Avril 2003. \\
\hline $\begin{array}{l}\text { Johnson, Derek } \\
\text { (États-Unis, 1976) }\end{array}$ & Frozen Light (2004) & Ensemble instrumental & $\begin{array}{l}\text { Montréal, NEM. Novembre } \\
2004 .\end{array}$ \\
\hline Kessler, Thomas (Suisse, 1937) & Dichterlesung (2002) & $\begin{array}{l}\text { Voix, objets divers, } \\
\text { électronique, théâtralité }\end{array}$ & $\begin{array}{l}\text { Montréal, Concerts M. Février } \\
2004 .\end{array}$ \\
\hline Kuljeric, Igor (Croatie, 1938) & Animal Farm (2003) & Opéra & Zagreb, Biennale. Avril 2003. \\
\hline $\begin{array}{l}\text { La Monte Young } \\
\text { (États-Unis, 1935) }\end{array}$ & $\begin{array}{l}\text { Just Charles and Cello in the } \\
\text { Romantic Chord (2002-2003) }\end{array}$ & $\begin{array}{l}\text { Violoncelle, vidéo, } \\
\text { électronique }\end{array}$ & Paris, CCMIX. Novembre 2003. \\
\hline Lanza, Mauro (Italie, 1975) & Barocco (1998-2003) & Voix et instruments d'enfants & Paris, Ircam. Février 2004. \\
\hline $\begin{array}{l}\text { Laporte, Jean-François } \\
\text { (Canada, 1968) }\end{array}$ & Confidence (2000) & Violon solo amplifié & $\begin{array}{l}\text { Montréal, Université de } \\
\text { Montréal. Janvier } 2000 .\end{array}$ \\
\hline Nordin, Jesper (Suède, 1971) & Vintage (2003) & $\begin{array}{l}\text { Percussion et traitement } \\
\text { électronique en temps réel }\end{array}$ & Paris, Ircam. Octobre 2003. \\
\hline Nova, Ricardo (Italie, 1960) & East Aka West (2002) & $\begin{array}{l}\text { Grand ensemble, chœur, } \\
\text { vidéo, électronique }\end{array}$ & Zagreb, Biennale. Avril 2003. \\
\hline Niblock, Phill (Etats-Unis, 1933) & Sweet Potato (2001) & Clarinette et électoacoustique & Genève, Archipel. Avril 2003. \\
\hline Parra, Hector (Espagne, 1976) & Strette (2003) & $\begin{array}{l}\text { Voix, projections visuelles, } \\
\text { traitement électronique en } \\
\text { temps réel }\end{array}$ & Paris, Ircam. Octobre 2003. \\
\hline Pelletier, Marie (Canada, 1959) & Dehors/Dedans (2002) & $\begin{array}{l}\text { Quatuor de saxophones } \\
\text { (théâtre musical) }\end{array}$ & Montréal, Quasar. Mai 2002. \\
\hline $\begin{array}{l}\text { Posadas, Alberto } \\
\text { (Espagne, 1967) }\end{array}$ & $\begin{array}{l}\text { Ondulado tiempo sonoro } \\
(2003)\end{array}$ & $\begin{array}{l}\text { Quatuor à cordes et } \\
\text { électronique }\end{array}$ & Paris, Ircam. Juin 2004. \\
\hline $\begin{array}{l}\text { Räisänen, Tomi } \\
\text { (Finlande, 1976) }\end{array}$ & Gatekeepers (2003) & Accordéon, sheng & $\begin{array}{l}\text { Abbaye de Royaumont } \\
\text { (France). Octobre } 2003 .\end{array}$ \\
\hline $\begin{array}{l}\text { Roedelius, H. Joachim } \\
\text { (Allemagne, 1934) }\end{array}$ & Happy Kitchen (1994) & $\begin{array}{l}\text { Instruments de cuisine, piano, } \\
\text { saxophone, électo. }\end{array}$ & Zagreb, Biennale. Avril 2003. \\
\hline $\begin{array}{l}\text { Sellars, James } \\
\text { (États-Unis, 1940) }\end{array}$ & Go (1997) & Ensemble instrumental (6) & Zagreb, Biennale. Avril 2003. \\
\hline Torre, Laurent (France, 1975) & Duo Concertante (2004) & Ensemble instrumental & Montréal, NEM. Novembre 2004. \\
\hline User (The) (Collectif, Canada) & Silophone (2000-2003) & $\begin{array}{l}\text { Silo à grains et électronique } \\
\text { (in situ) }\end{array}$ & $\begin{array}{l}\text { Montréal. Divers événements. } \\
\text { 2000-2003. }\end{array}$ \\
\hline
\end{tabular}

